\title{
Fluorescence Talbot microscope using incoherent source
}

Yangyang Sun

Shuo Pang 


\title{
Fluorescence Talbot microscope using incoherent source
}

\author{
Yangyang Sun and Shuo Pang* \\ University of Central Florida, Optical Imaging System Laboratory, The College of Optics and Photonics, 4304 Scorpius Street, Orlando, \\ Florida 32816, United States
}

\begin{abstract}
Fluorescence Talbot microscope is a scalable field-of-view (FOV) imaging platform, which takes advantage of the phase sensitivity of the self-image of a periodic structure. Such a system can maintain the microscopic resolution and extend the FOV for the whole slide $(15 \mathrm{~mm} \times 15 \mathrm{~mm})$ scanning. Previously reported Talbot fluorescence systems, tabletop and on-chip device alike, rely on the coherence of the illumination source, limiting their potential applications in low-resource setting environment. A more cost-effective setup using a light-emitting diode, which has an area of $4 \mathrm{~mm}^{2}$ and a full width at half maximum of $16 \mathrm{~nm}$ in wavelength, is demonstrated. Compared to the illumination that is spatially filtered by a single pinhole, our system has achieved an illumination intensity that is 357 times higher. The reconstructed image quality is comparable to that of a $10 \times$ microscope objective. Various samples, such as fluorescent beads, green fluorescence protein-labeled HeLa cells, and a mouse kidney slide, were reconstructed by the system. @ 2016 Society of Photo-Optical Instrumentation Engineers (SPIE) [DOI: 10 .1117/1.JBO.21.8.086003]
\end{abstract}

Keywords: Talbot effect; Lau effect; microscopy; fluorescence.

Paper 160206R received Mar. 31, 2016; accepted for publication Jul. 20, 2016; published online Aug. 8, 2016.

\section{Introduction}

A large field-of-view (FOV) microscopy is often desirable for imaging the migration and division of a large cell population. ${ }^{1,2}$ Conventional microscope objective lens with a decent fluorescence collection efficiency $[20 \times$, numerical aperture $(\mathrm{NA})=$ 0.5 ] have a limited FOV of $<1 \mathrm{~mm}^{2}$. To increase the FOV, the sample is typically transported with a translational stage which increases the cost and complexity of the system. Mechanical movement also limits the acquisition rate. A wide-field microscope system based on microcamera array can capture an image with a resolution better than $3 \mu \mathrm{m} .{ }^{3}$ The approach, named multiscale lens design, divides the large FOV using small-scale lens and camera arrays; it can greatly improve the space-bandwidth limit of the optical design with single optical axis. Fluorescence Talbot microscope (FTM), also using array elements to achieve scalable FOV imaging, has been developed, which has high collection efficiency and a large FOV of $\sim 14 \mathrm{~mm}^{2}$ with a resolution of $1.1 \mu \mathrm{m} .{ }^{4,5}$ FTM generates a self-imaging periodic structure, which serves as an illumination pattern at the Talbot distance away from the original structure. Talbot illumination has a longer working distance and an improved phase sensitivity, which makes small angular scan possible. ${ }^{6,7}$ A low-cost, chipscale microscopic imaging device equipped with an MEMS mirror based on FTM method has been demonstrated. ${ }^{4}$

The aforementioned systems all use laser as illumination source, due to the requirement of the coherence of self-imaging phenomena. The cost and form factor of a laser with highquality factor beam limit the applications of FTM in the lowesource setting environment. In this paper, we proposed a setup using the so-called Talbot-Lau interferometer ${ }^{8-10}$ to address this issue. We use two sets of microlens arrays (MLA) and a lightemitting diode (LED) with a dimension of $2 \mathrm{~mm} \times 2 \mathrm{~mm}$ to generate Talbot illumination patterns. We demonstrated the imaging capability of such a setup by imaging fluorescent beads, green fluorescence protein-labeled HeLa cells, and a mouse kidney slide.

The paper is organized as follows. In Sec. 2, we first explain the imaging principle of the setup using incoherent light source. In Sec. 3, we describe our system setup and compare the point spread function (PSF) of experimental results with our simulation results. We also discuss the advantage of the setup in illumination efficiency. In Sec. 4, the experimental imaging results are presented and compared with a $10 \times$ microscope. Finally, we conclude our work in Sec. 5.

\section{Imaging Principle}

The self-imaging phenomena of periodic intensity pattern, known as the Talbot effect, have been extensively investigated under various circumstances ${ }^{11-13}$ and in different wavelength regimes. ${ }^{14}$ The Talbot effect requires spatial and temporal coherence of the source and, hence, most systems use laser as the light source. The Talbot effect, in essence, is a two-dimensional (2-D) periodic field repeating itself periodically along the wave propagation direction. This period in the propagation direction is called the Talbot distance, $D_{T}$

$D_{T}=\frac{2 d^{2}}{\lambda}$

where $d$ is the period of the field and $\lambda$ is the wavelength of the light source. To generate Talbot illumination with a spatially incoherent source, one simple solution is adding a pinhole to gain the spatial coherence, as shown in Fig. 1(a). When the pinhole shifts within the plane, as shown in Fig. 1(b), the Talbot 
(a)

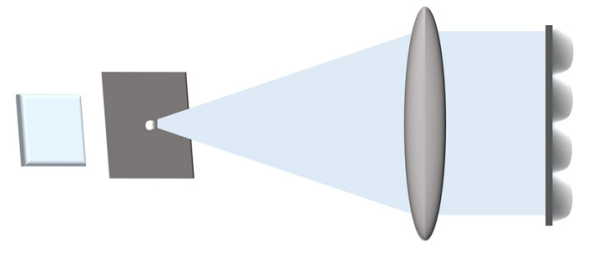

(b)

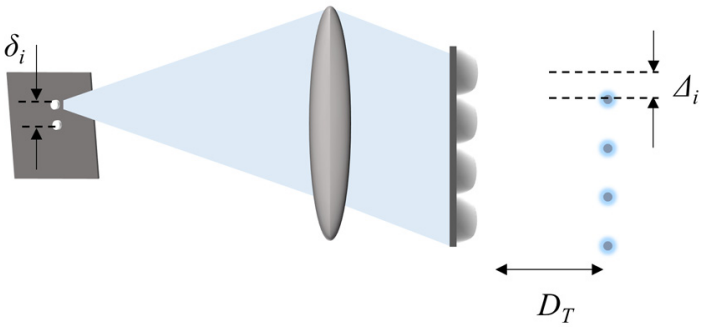

(c)

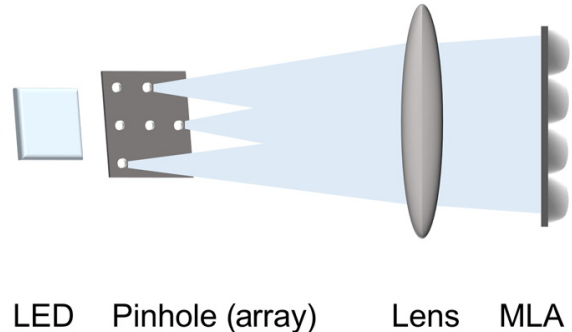

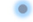

$\bullet$

-

-

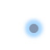

$\bullet$

$\bullet$

-

$\bullet$

Fig. 1 (a) Configuration of the Talbot effect with a single pinhole. (b) The shifted pinhole results in a shifted Talbot grid. (c) Using a pinhole array, whose period follows Eq. (3), can achieve same high contrast Talbot grid as one pinhole and higher illumination efficiency.

grid shifts correspondingly. If the displacement of the Talbot grid is a multiple of the period of the grid, the shifted Talbot grid will coincide with the original Talbot grid. ${ }^{9}$ Therefore, we can add in a pinhole array to replace a single pinhole, as shown in Fig. 1(c). Each pinhole generates a shifted version of the original Talbot grid. The sample is placed at one Talbot distance, $D_{T}$, away from the focal plane of the MLA, which is referred as the Talbot plane. The following discussion is in one dimensional situation, which can be easily expanded to 2-D situations. Under the paraxial approximation, the Talbot grid generated by the $i$ 'th pinhole has a lateral displacement

$\Delta_{i}=\frac{D_{T}}{f} \delta_{i}$,

where $\delta_{i}$ is the distance between the $i$ 'th pinhole and the central pinhole and $f$ is the focal length of the collimation lens. The total irradiance pattern is the summation of all shifted Talbot grids, which can be expressed as

$T(x)=\sum_{i} T_{0}\left(x-\Delta_{i}\right)$,

where $T_{0}$ is the Talbot grid generated by the central pinhole and $\Delta_{i}$ is the displacement of the Talbot grid generated by the $i$ 'th pinhole. Since $T_{0}$ is an infinite periodic pattern, $T$ will have the same pattern as $T_{0}$, as long as the $\Delta_{i}$ satisfies

$\Delta_{i}=n p_{2} ; \quad n=0,1,2,3 \ldots$,

where $p_{2}$ is the pitch of the MLA. This is the condition that the Talbot grids generated by different pinholes coincide with each other at Talbot plane.

\section{Materials and Methods}

The experimental setup is shown in Fig. 2. We employ another MLA (MLA1 in Fig. 2) to concentrate the light onto the pinhole array (HTA Photomask) to improve the illumination efficiency, which is similar to the microlens setup in the spinning disk (Nipkow Disk) confocal microscope. The pitch of MLA1 (SUSS MicroOptics) is $100 \mu \mathrm{m}$ with an NA of 0.17 . The diameter of individual pinhole on the pinhole array is $20 \mu \mathrm{m}$ and the period is $100 \mu \mathrm{m}$, matching that of the MLA1. The pinhole array is placed in the front focal plane of the lens L1 ( $f_{1}=50 \mathrm{~mm}$, Newport). The lenses L1 and L2 relay the grid to the front focal plane of the lens L3 with the exact magnification so that the condition of Eq. (3) is satisfied. A piezodriven mirror placed at the back focal plane of the lens L1 for sample scanning. The mirror is driven by a piezo-motor (AGM100L, Newport). The mirror is relayed to the MLA2 by L2 ( $f_{2}=100 \mathrm{~mm}$, Newport) and L3 (focal length 18 to $35 \mathrm{~mm}$, Nikon AF) to minimize beam walking. The pitch of MLA2 (SUSS MicroOptics) is $30 \mu \mathrm{m}$ with an NA of 0.15 . The lateral magnification by the lens L1 and lens L2 is 2 . Given the size of individual pinhole, the calculated spot size is $5.8 \mu \mathrm{m}$, which is same as our measurement result. The individual pinhole size can be selected according to applications; smaller pinhole diameter will result in a higher spatial resolution with compromised illumination efficiency. The sample is placed at Talbot plane, $3.77 \mathrm{~mm}$ away from the focal plane of the MLA2. The detection system consists of an objective $\left(f_{o}=50 \mathrm{~mm}\right.$, $\mathrm{NA}=0.13$, Nikon), a tube lens $\left(f_{e}=200 \mathrm{~mm}\right.$, Newport), an emission filter, and a camera (Manta G-145B, AVT). The illumination grid on the sample plane probes an array of spots on the fluorescent sample whose fluorescence image is captured by the detector. The motor-driven mirror scans the illumination grid over the sample to form a stack of frames captured by the camera. We divide the detector into small sensor blocks according to the illumination grid and integrate the gray value within the blocks to represent the value on the probed points on the sample. With the probe of illumination grid scanning over the sample, we could reconstruct the image of fluorescence sample. This processing procedure is the same as the one in FTM. ${ }^{2}$ The storage of the three-dimensional data cube takes large memory space given the number of scanning steps and the pixel number of each frame. In our experiment, the data cube contains more than 10 thousand frames and each frame is about 4 megabytes. To reduce the data storage and improve the acquisition rate, we operate the detector on a $3 \times 3$ binning mode. It is worth noting that the resolution of the final image depends on the spot size of the illumination grid instead of the pixel size of the detector. We did not use the binning mode larger than $3 \times 3$ because of the cross talk.

To quantify the performance of our imaging system, we record the PSF of different illumination configurations, including (1) an LED illuminating the MLA, (2) an LED focused onto a single pinhole, (3) the proposed setup with an LED (UHP-T460-EP Prizmatix) and a pinhole array, and (4) a 488 laser source (OBIS, Coherent). The schematics and the images of the Talbot grids are shown in Figs. 3(a)-3(d). Figure 3(a) shows the pattern at Talbot plane when an LED is used to directly illuminate the MLA. In the recorded image, the grid pattern cannot be discerned from the background. On the contrary, as shown in Fig. 3(d), in which the 488-nm laser is the light source, a Talbot grid with spot diameter $<2 \mu \mathrm{m}$ can be observed, owning to its high temporal and spatial coherence. 


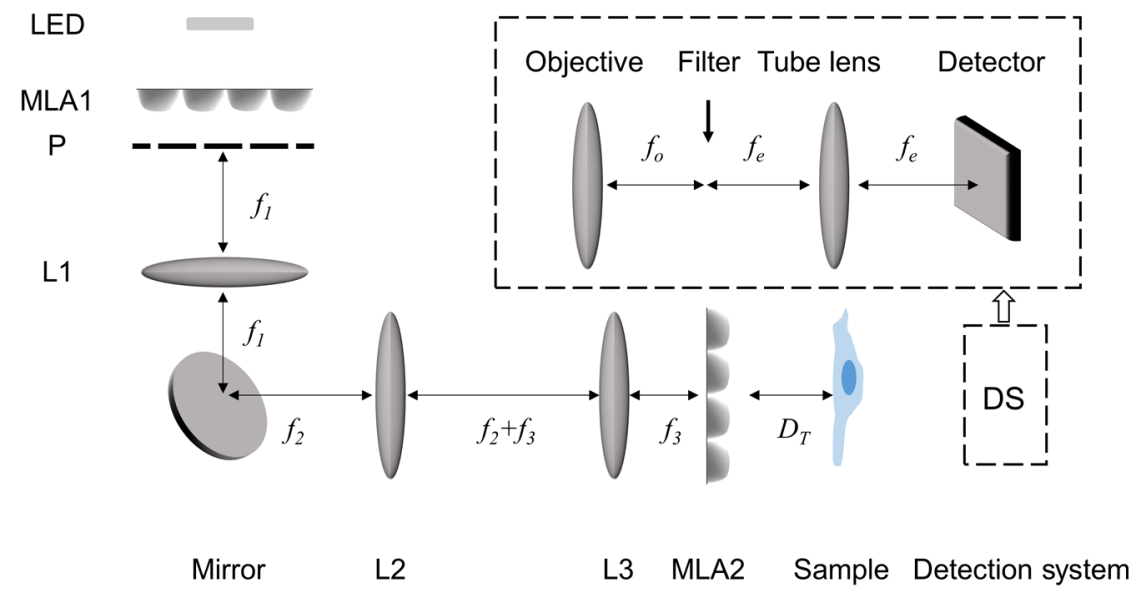

Fig. 2 The configuration of the incoherent Talbot microscopy system. A microlens array (MLA1) is placed after an incoherent light source (LED). The pinhole array, $P$, is placed at the focal plane of the MLA1. Two lenses $L 1$ and $L 2$, relay the focal grid to the front focal plane of the projection lens $L 3$. $L 2$ and $L 3$ relay the piezo-driven mirror to the imaging microlens array (MLA2). The sample is placed at one Talbot distance away from the focal plane of MLA2. The illumination Talbot grid on the sample plane excites the fluorescent sample and the signal is captured by a $4 \times$ detection system. The detection system consists of an objective, an emission filter, a tube lens, and a detector (DS).

To improve the spatial coherence, the common solution is to focus the LED onto a single pinhole, as shown in Fig. 3(b). We use a $40 \times$ objective with an NA of 0.75 to focus the light. With a $40-\mu \mathrm{m}$ equivalent pinhole size after the magnification of L1 and L2, the measured Talbot spot has a diameter of $5.8 \mu \mathrm{m}$. The maximum illumination power by the system depends on the product of the solid angle of the collimation lens L1 and the pinhole size, characterized by the illumination etendue, which is determined by the Lagrange invariant of the illumination system. The illumination efficiency of a single pinhole [Fig. 3(b)] is the ratio between the etendue of the LED and the illumination etendue, which is independent on the focusing power of the objective. The MLA-condensed pinhole array partitions the Lagrange invariant of the source into small fragments, as shown in Fig. 3(c). The Lagrange invariant of each microlens before the pinhole array is similar to that of the collection system. The Lagrange invariant of the LED is four orders of magnitude larger than that of a single pinhole system; hence, the major limitation is the effective illumination area of the pinhole array. In other words, the number of the individual pinholes that can be collected determines the illumination efficiency of the proposed method. The improvement of the illumination efficiency can be calculated as $N / q^{2}$, where $N$ is the number of the pinholes within the effective illumination area of the LED and $q$ is the ratio between the NA of MLA1 and that of the lens L1. In our setup, $N$ is $~ 900$, and the NA of L1 is 0.12 and the NA of MLA1 is 0.17 . Therefore, the calculated improvement is $\sim 440$. The measured improvement of illumination efficiency is 357 . The difference could be attributed to the misalignment and aberrations of the lenses. Figure 4 shows the cross-section profile of the Talbot grid. The absolute intensities of the profiles are normalized to their exposure time. To better illustrate the difference of the intensity, we scale up the profile of the single pinhole setup 100 times. We also simulate the Talbot profile as the black curve shown in Fig. 4. The simulated profile has a full width at half maximum (FWHM) of $5.8 \mu \mathrm{m}$.

Temporal coherence also has an impact on the system resolution. We measured the spectrum of the laser and the LED with an excitation filter $(480 / 30 \mathrm{~nm})$ as shown in Fig. 3(e). (a)
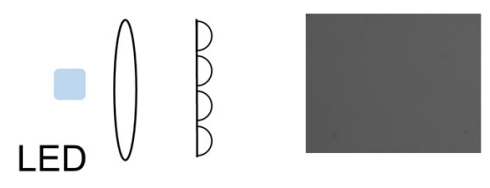

(b)
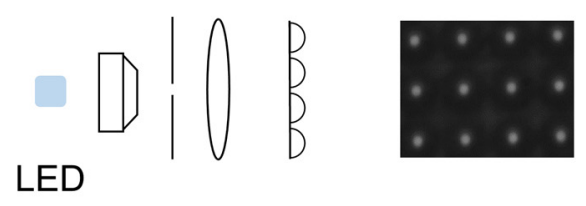

(c)
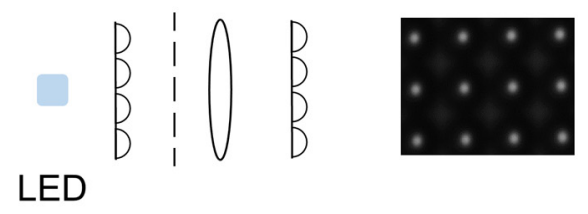

(d)

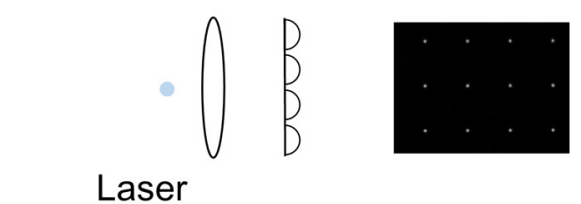

(e)

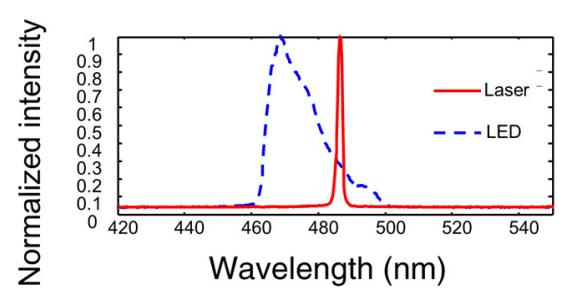

Fig. 3 The images of the Talbot grids of the MLA illuminated by (a) an LED, (b) a $40 \times$ objective and a pinhole, (c) an MLA and a pinhole array, and (d) a laser. (e) Normalized spectrum distribution of the LED and the laser. Blue curve is the spectrum of LED and red curve is that of the laser. The profiles are normalized to their peaks. 


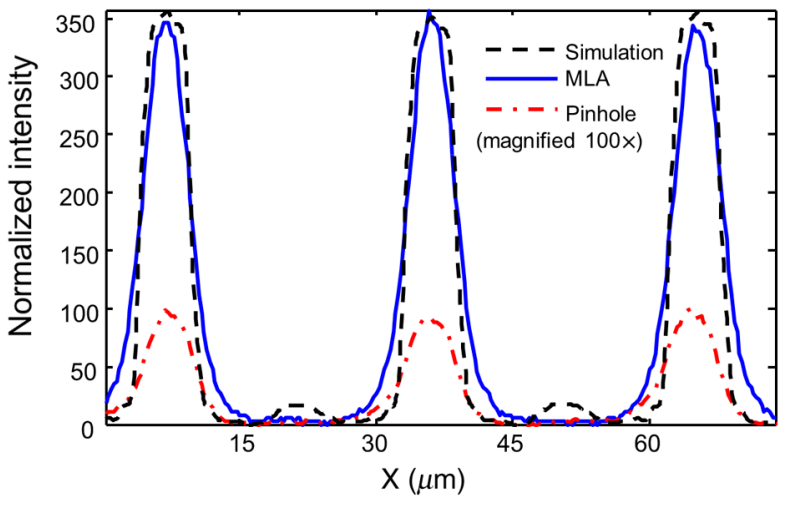

Fig. 4 The simulated and experimental profiles of Talbot grids. The black curve is the simulated profile of the Talbot spots. The blue curve is the profile of the grid of our proposed setup and the red curve is that of the one pinhole setup. The experimental profiles are first normalized to their exposure time. Then the Talbot profile from a single pinhole (red curve) is magnified 100 times.

The bandwidth of the filtered LED is $16 \mathrm{~nm}$ while the bandwidth of the laser is $1.8 \mathrm{~nm}$. It is worth mentioning that the location of the Talbot plane varies with the wavelength as $D_{T} \Delta \lambda / \lambda_{c}$, where $\lambda_{c}$ is the central wavelength of the source and $\Delta \lambda$ is the deviation from the central wavelength. The condition of Eq. (3) can be satisfied at only one wavelength. The deviation of the Talbot plane results in a mismatch of the period of the pinhole array and the Talbot distance. In other words, the Talbot grids by all pinholes coincide well on the Talbot plane only at the central wavelength; the broadband of the source also contributes to the broadening of the PSF.

\section{Results and Discussions}

To examine the performance of our imaging system on fluorescent samples, we first image fluorescent microspheres $(d=$ $15 \mu \mathrm{m}$, FS07F, Bangs Laboratories), which are air-dried on a microscope slide. We insert a fluorescence emission filter (D535/40, Chroma) in the detection path. The reconstructed images are shown in Figs. 5(a) and 5(b). The scanning step is $0.5 \mu \mathrm{m}$.

To demonstrate the performance of the system on the biological sample, we image fluorescent HeLa cell sample. The reconstructed images are shown in Figs. 5(c) and 5(d). In addition, a mouse kidney slice (Life Technologies) sample is also imaged by the system. We use a damped Richardson-Lucy deconvolution on the reconstructed images ${ }^{15}$ to improve the contrast. This is a blind deconvolution approach, which does not require any explicit knowledge of the PSF. The reconstructed images are shown in Figs. 6(c) and 6(d) and are compared with the image under the conventional $10 \times$ fluorescence microscope as shown in Figs. 6(a) and 6(b). The image quality of the reconstructed image is comparable to the $10 \times$ fluorescence microscope with a larger FOV. The FOV of the reconstructed image is $1.43 \mathrm{~mm} \times$ $1.16 \mathrm{~mm}$ limited by the optics elements in the detection path. The FOV could be scaled up with larger size MLA and the objective with larger FOV. For conventional objective used in microscope, it is difficult to achieve large FOV and high NA at the same time. In our system, the resolution depends on the spot size of illumination grid instead of the image quality on the detector. Thus, our system is of higher tolerance of the aberrations in the optics elements in the detection system. One could choose the lens with large FOV and high NA in the detection system while keeping the same resolution. A $14-\mathrm{mm}^{2}$ FOV system with similar detection configurations has been reported in Ref. 2. Another advantage is that the acquisition time is independent on the FOV. The mechanical angular movement for one step takes $0.1 \mathrm{~s}$. The step size is around $0.3 \mu \mathrm{m}$. In our setup, we scan $1.5 \mathrm{~mm}^{2}$ within $18 \mathrm{~min}$. It is worth noting that the scanning time stays the same in larger FOV applications. Thus, the scanning rate could be improved with the FOV.

Different from the bright-field imaging, the resolution of the reconstructed fluorescence image depends not only on the pinhole size of the pinhole array but also the alignment. It is convenient to align the system well on the central wavelength of the LED. In bright-field imaging, the FWHM of the PSF is a good estimation of the lateral resolution of our system and the system can be aligned well on the central wavelength of the LED. However, for fluorescence imaging, the system should be aligned well on the peak of the excitation spectrum of the fluorescent sample. If the excitation peak has a relative large deviation from the LED emission peak, the alignment to the excitation peak would be more difficult, resulting in an inferior PSF. Although our incoherent source-based system cannot outperform the laser-based system on the aspect of resolution, our system has three major advantages compared to other scanning optical microscope. First of all, the cost of the light source is reduced. Compared with a 488-nm laser with maximum power of $100 \mathrm{~mW}$, our system with bright LED has a maximum power of $4 \mathrm{~W}$, and its price is only about a quarter
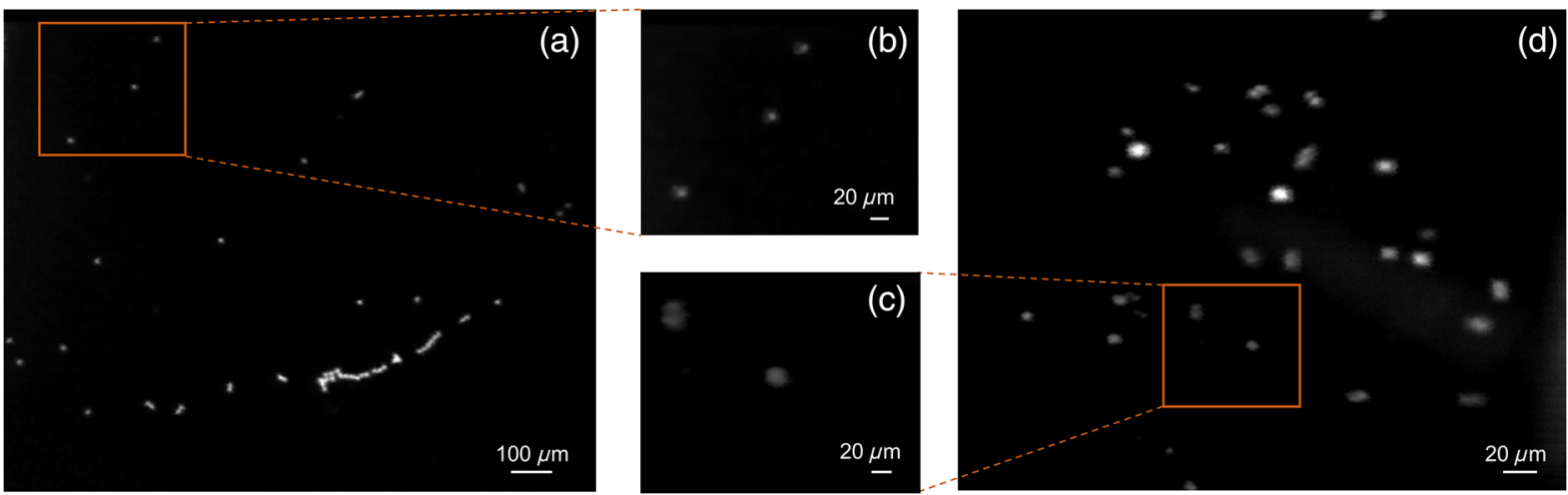

Fig. 5 The reconstructed images of microspheres and HeLa cells. (a) and (b) The reconstructed images of fluorescent microspheres, (b) is a close-up image of (a). (c) and (d) The reconstructed images of HeLa cells, (c) is a close-up image of (d). 

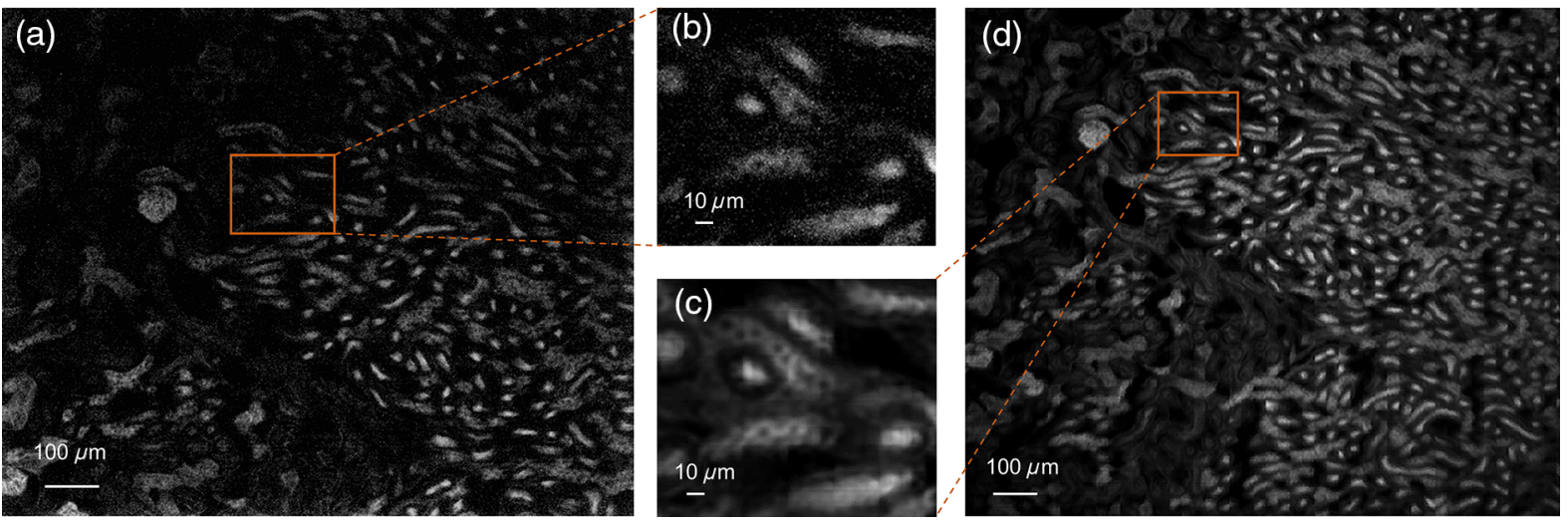

Fig. 6 The mouse kidney slide under fluorescence microscope and our imaging system: (a) and (b) The microscope image under conventional $10 \times$ fluorescence microscope. (b) is a close-up image of (a). (c) and (d) The reconstructed image of the incoherent Talbot microscopy system with damped RichardsonLucy deconvolution. (c) is a close-up image of (d).

of that of laser. This advantage becomes more significant when the imaging requires even higher excitation power. Second, the FOV of our system is scalable with MLA of larger dimension. Third, the scanning time is independent of the FOV. Thus, the acquisition rate is more advantageous in large FOV imaging. In addition, the pattern generated by the incoherent source is free of speckles, which provides a uniform illumination.

\section{Conclusions}

In summary, we have demonstrated an incoherent illuminated FTM with the Talbot-Lau effect. Different from the conventional laser illuminated scanning microscope, the proposed setup using an inexpensive and spatially incoherent LED source costs one order of magnitude less. In addition, the resultant images are free from interference speckles. Compared with the multicamera array system, ${ }^{3}$ our system benefits from the inexpensive scalability and the potential of on-chip microscopy implementation. The use of a pinhole array gives an improvement of 357 times in illumination efficiency compared with the single pinhole setup. The FWHM of PSF of our setup is $\sim 6 \mu \mathrm{m}$, which is the same as the single pinhole setup. We have applied our system in imaging various fluorescent samples. The system has the potential in simple multicolor fluorescence imaging with minor adjustment in alignment.

\section{Acknowledgments}

The authors would like to thank Professor Karl X. Chai and Dr. Limei Chen from the Burnett School of Biomedical Sciences for the HeLa cell samples.

\section{References}

1. A. A. Cohen et al., "Dynamic proteomics of individual cancer cells in response to a drug," Science 322(5907), 1511-1516 (2008).

2. Y. E. Kim et al., "Engineering a polarity-sensitive biosensor for timelapse imaging of apoptotic processes and degeneration," Nat. Methods 7(1), 67-73 (2010).
3. D. L. Marks et al., "Wide-field microscopy using microcamera arrays," Proc. SPIE 8589, $85890 \mathrm{Z}$ (2013).

4. S. Pang et al., "Wide and scalable field-of-view Talbot-grid-based fluorescence microscopy," Opt. Lett. 37(23), 5018-5020 (2012).

5. S. Pang et al., "Wide field-of-view Talbot grid-based microscopy for multicolor fluorescence imaging," Opt. Express 21, 14555 (2013).

6. Y. Sun and S. Pang, "Multi-perspective fluorescence Talbot microscopy," in Frontiers in Optics, Paper No. FTh3G-6, Optical Society of America, San Jose (2015).

7. Y. Sun and S. Pang, "Multi-perspective scanning microscope based on Talbot effect," Appl. Phys. Lett. 108(2), 021102 (2016).

8. E. Lau, "Beugungserscheinungen an doppelrastern," Ann. Phys. 437(7-8), 417-423 (1948)

9. J. Jahns and A. W. Lohmann, "The Lau effect (a diffraction experiment with incoherent illumination)," Opt. Commun. 28(3), 263-267 (1979).

10. K. Patorski and P. Szwaykowski, "Optical differentiation of quasi-periodic patterns using Talbot interferometry," Opt. Acta Int. J. Opt. 31(1), 23-31 (1984).

11. Y. Sun et al., "Talbot interferometry with curved quasi-periodic gratings: towards large field of view X-ray phase-contrast imaging," Opt. Express 23(20), 26576-26585 (2015).

12. S. De Nicola et al., "Talbot self-image effect in digital holography and its application to spectrometry," Opt. Lett. 29(1), 104-106 (2004).

13. A. W. Lohmann and J. A. Thomas, "Making an array illuminator based on the Talbot effect," Appl. Opt. 29(29), 4337-4340 (1990).

14. A. Momose et al., "Phase-contrast X-ray computed tomography for observing biological soft tissues," Nat. Med. 2(4), 473-475 (1996).

15. R. L. White, "Image restoration using the damped Richardson-Lucy method," Proc. SPIE 2198, 1342-1348 (1994).

Yangyang Sun is a graduate student at the University of Central Florida. He received his BS degree in information engineering from Nanjing University in 2013. His current research interests include computational imaging and machine vision.

Shuo Pang is an assistant professor in the College of Optics and Photonics at the University of Central Florida. His research focuses on computational imaging in both visible and $\mathrm{x}$-ray regimes, with applications in medical imaging and biological research. He obtained his $\mathrm{MS}$ and $\mathrm{PhD}$ degrees in electrical engineering from California Institute of Technology (Caltech), and the BS degree in optical engineering from Tsinghua University. 